\title{
A Study of Denotative Similarity with Restricted Word Associations ${ }^{1}$
}

\author{
Charles A. Perfetti ${ }^{2}$ \\ University of Michigan, Ann Arbor, Michigan 48104
}

\begin{abstract}
Data for this study were collected in two parts. In the first, $70 \mathrm{Ss}$ responded to a list of stimuli in seven tasks of restricted association. The second part of the study employed stimuli consisting of 11 of these original stimuli plus their primary responses in each of the seven tasks. These were given to 50 new Ss under the same seven task instructions. The seven tasks were Superordinates, Coordinates, Similars, Contrasts, Functions, Qualities, and Parts. The main analysis of data was the derivation of matrices of response overlap for the seven semantic relationships corresponding to the restricted-association tasks.

Primary interest centered on similarity relationships. High overlap along the diagonal of the response-overlap matrix characterized the Similars. Overlap in five of these diagonal cells was found to discriminate Similars from the other relationships. A measure of the degree of Restricted-Association Similarity (RAS) was derived, based on this discriminating overlap.
\end{abstract}

This paper describes an exploratory attempt at a methodology for the study of word similarity. Similarity, which has achieved important status as an independent variable in verbal learning, has been previously measured by rating scales (Haagen, 1949), connotative distances based on the semantic differential (Osgood, Succi, and Tannenbaum, 1958), and various indexes of association and associative overlap (Marshall and Cofer, 1963). Several such measures of similarity have been studied by Wimer (1963) who factorially analyzed some of their interrelationships.

${ }^{1}$ The author wishes to acknowledge the helpful comments of Dr. Frank Koen, and, especially, the extensive assistance of Dr. Klaus Riegel on an earlier version of this paper. This research was jointly supported by the author's predoctoral fellowship from the United States Public Health Service and by NIMH grant MH 07619 .

${ }^{2}$ Now at University of Pittsburgh, Pittsburgh, Pa. 15213.
The present approach combines a measure of restricted-association overlap, similar to previous measures of free-association overlap, with a conception of meaning which relies on formal and physical semantic relationships in a manner previously suggested by Riegel and Riegel (1963). The basic assumption of the approach is that the similarity of a pair of words can be estimated by the degree to which they share the same semantic relationships with the same words-or, operationally, the degree to which they share a distribution of restricted-association responses. Tasks of restricted association are assumed to correspond to various semantic relationships between words. Seven of the 16 tasks used by Riegel (1965a) were chosen for this purpose: Superordinate, Coordinate, Similar, Contrast, Part, Function, and Quality. Each task describes the relationship of the response to the stimulus. The degree to which a pair of words has overlapping re- 
sponses under each of these tasks of restricted association is considered an index of their denotative similarity. Thus two words which are perfectly similar are assumed to have the same Superordinates, the same Coordinates, Parts, etc.-i.e., $100 \%$ overlap or completely identical, response distributions under the seven tasks. The degree of similarity can therefore be estimated by the deviation of their response distributions from perfect overlap.

While the use of this overlap method as a measure of similarity was the main purpose of the study, there was a secondary interest in observing whether words bearing relationships other than similarity, e.g., superordination, would produce a unique pattern of response overlap:

\section{Method}

\section{Restricted-Association Tasks}

Seven tasks of restricted associations were presented with the following instructions to Ss:

Superordinates: Find a class name for the stimulus. For instance class names for the word FORK are SILVERWARE or UTENSIL. Class names for the word LIMOUSINE are CAR or VEHICLE.

Coordinates: Find another member of the class to which the stimulus belongs. For instance, SPOON or KNIFE belong to the same class as the word FORK. TRAIN or BIKE belong to the same class as CAR.

Parts or Attributes: Find a word that denotes an essential part or attribute of the stimulus. For instance, essential parts of a FORK are the HANDLE or the $M E T A L$. Essential attributes of WISDOM are EXPERIENCE or MATURITY.

Similar: Find a word that means essentially the same as the stimulus. For instance $R A K E$ or BRANCH may be regarded as similar to FORK. AUTO or AUTOMOBILE may be regarded as similar to $C A R$.
Contrasts: Find a word that means essentially the opposite of the stimulus. For instance, KNIFE or SPOON may be regarded as contrasts to FORK. FOOLISHNESS or STUPIDITY may be regarded as contrasts to WIS$D O M$.

Functions: Find a word that denotes a usage of the stimulus. For instance, a FORK is used to EAT or TAKEUP. CAR is used to TRAVEL or DRIVE.

Qualities: Find a word that denotes a quality of the stimulus. For instance, a FORK is POINTED or HEAVY. A CAR is FAST and SHINY.

\section{Subjects and Procedure}

Part 1. Seventy Ss enrolled in an introductory psychology course at The University of Michigan served in the first part of the study. Each $S$ was given an instruction sheet and seven stimulus sheets, one for each task described above. Each sheet contained the same 50 stimuli, all of which were nouns. The order in which $S$ s received the seven tasks was randomized. The Ss were instructed to respond to one task at a time, without referring to their responses on previous pages. They gave one response to each stimulus on each page. The responses thus collected were then used in Part 2 of the study.

Part 2. Fifty Ss enrolled in introductory psychology courses at Michigan served in the second part of the study. The stimuli were chosen from the first part of the study in the following manner: Eleven of the original 50 stimuli, plus the primary responses to these 11 stimuli under each of the seven tasks, were selected and are listed in Table 1. After the eliminations of duplicated responses, the total number of stimuli for the second part of the study was 54 . The administration of the tasks followed the same procedure as described for Part 1.

\section{Results AND Discussion}

Part of the aim of the study was to derive matrices of response overlap, based on 11 stimuli for each of the seven word relations. Each of the seven relationships was expected to produce a unique overlap matrix. In particular, for Similars, a matrix of response overlap with the seven tasks 
PERFETTI

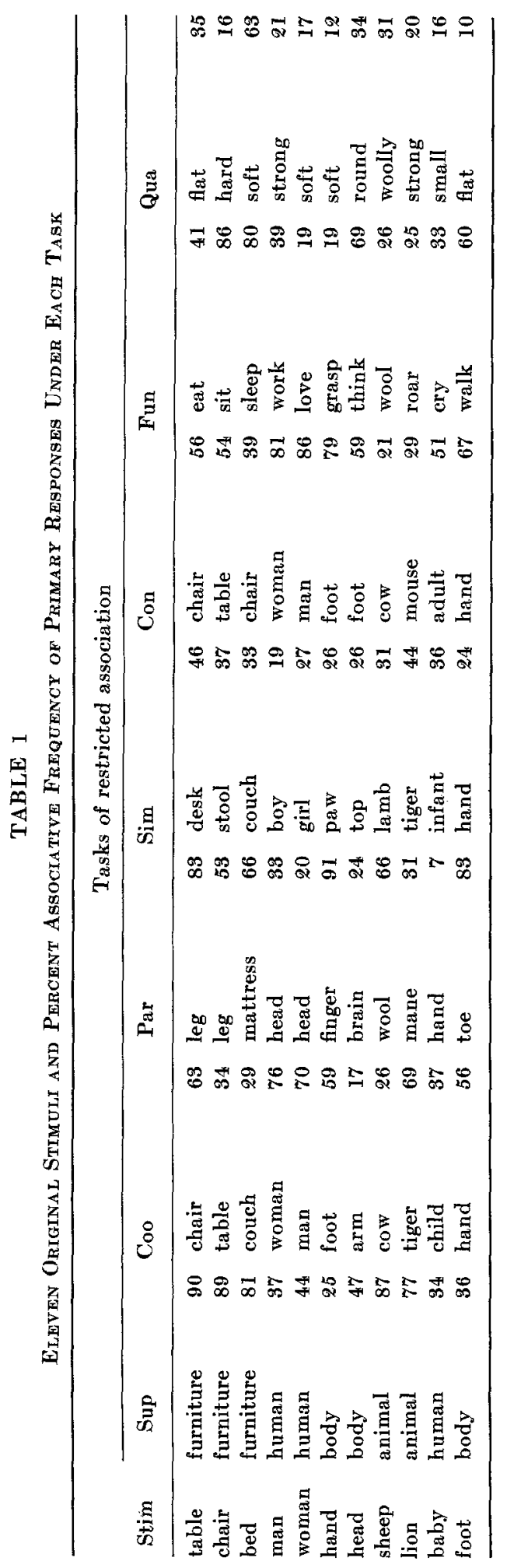


occupying corresponding rows and columns, was expected to show a pattern of high overlap along its diagonal-reflecting the assumption that similar words should have the same Superordinates, Parts, etc.

\section{An Example of Response Overlap for Two Similars}

Clarification of the method of analysis can be served by a consideration of the primary similars, TABLE and DESK. Under the Superordinate task there was one response shared by these two stimuli-FURNITURE, which was given to TABLE by $49 \mathrm{Ss}$ and to DESK by $47 \mathrm{Ss}$. The smaller of these numbers, in this case 47 , was entered into the overlap matrix as a percentage, in this case $94 \%$. Table 2 gives the complete

\section{TABLE 2}

Overlap Matrix for Table-DesK in Percent

\begin{tabular}{lrrrrrrr}
\hline R & & & & & & & \\
\hline Sup & Sup & Coo & Par & Sim & Con & Fun & Qua \\
Coo & 94 & 6 & 0 & 8 & 0 & 0 & 0 \\
Par & 10 & 38 & 0 & 60 & 46 & 0 & 0 \\
Sim & 0 & 0 & 54 & 0 & 0 & 2 & 12 \\
Con & 6 & 38 & 4 & 64 & 12 & 0 & 4 \\
Fun & 0 & 30 & 0 & 18 & 58 & 0 & 0 \\
Qua & 2 & 2 & 6 & 6 & 0 & 34 & 4 \\
\hline & 2 & 2 & 16 & 2 & 0 & 4 & 42 \\
\hline
\end{tabular}

minimum overlap matrix thus derived for $T A B L E$ and DESK. As predicted for Similars, the highest overlap occurred on the diagonal. Among the cells off the diagonal, only those involving Coordinates show high overlap.

In the determination of the overlaps, a procedure employed by Deese (1962) for free associates was used, modified by the assumption that under the Similar instructions each stimulus evoked itself as its primary response. Thus, the number of times that one of the paired stimuli evoked the other, under any task instruction, was added to the overlap figures in the Similar column (or row) for that stimulus. Accordingly, the $60 \%$ overlap in cell (Coo, Sim) for TABLE$D E S K$ has two components: $12 \%$ of the Similars given to $T A B L E$ were given as Coordinates to $D E S K$; in addition, $D E S K$ itself was given as a Similar to TABLE $48 \%$ of the time (to be expected, of course, since TABLE and DESK are primary Similars). Cell (Sim, Coo) reflects the opposite situation: $16 \%$ of Coordinates to TABLE were Similars to DESK; plus, $22 \%$ of Ss gave TABLE as Similar to DESK. The above technique was applied to all stimuli across the Similar row and Similar column. For the cell (Sim, Sim) two figures of mutual evocation are obtained, e.g., TABLE was given as Similar to $D E S K$ by $22 \%$ of Ss, while DESK was given as Similar to TABLE by $48 \%$ of Ss. In these cases, the smaller of the two numbers was added, consistent with the technique of minimum overlap. The necessity for this procedure becomes apparent when the alternative of not adding mutual evocations is considered: If, in an extreme case, two words were so similar as to evoke only each other under the Similar instructions, their common overlap would necessarily be zero for both the Similar row and column.

\section{Mean Overlap Matrix for Similars}

For each relationship, a matrix of mean response overlap was constructed from the overlap matrices of each of the 11 word pairs representing a given relationship, as determined by the primary responses from Part 1. Although mean matrices for all seven relationships were involved in the subsequent analyses, only the matrix for Similars is given here (Table 3 ).

Matrices were expected to produce discriminable differences in overlap patterns among the various tasks. A nonmathematical cut-off technique was used to make such discriminations. Table 4 represents the "critical" cells-cells which were found

\section{TABLE 3}

Mean Overlap Matrix for Similars in Percent

\begin{tabular}{lrrrrrrr}
\hline S & & & & & & & \\
\hline Sup & Sup & Coo & Par & Sim & Con & Fun & Qua \\
Coo & 69 & 10 & 1 & 21 & 3 & 2 & 1 \\
Par & 9 & 21 & 2 & 54 & 13 & 1 & 1 \\
Sim & 2 & 3 & 30 & 3 & 1 & 7 & 9 \\
Con & 12 & 45 & 2 & 58 & 16 & 1 & 1 \\
Fun & 2 & 13 & 0 & 23 & 33 & 0 & 0 \\
Qua & 1 & 2 & 7 & 3 & 0 & 35 & 7 \\
\hline & 1 & 1 & 7 & 2 & 0 & 3 & 26 \\
\hline
\end{tabular}


TABLE 4

Cells in Which High Overla Distinguishes One or More Relationship from the Others

\begin{tabular}{|c|c|c|c|c|c|c|c|}
\hline $\mathrm{R} / \mathrm{S}$ & Sup & $\mathrm{CoO}$ & Par & Sim & Con & Fun & Qua \\
\hline Sup & $\begin{array}{l}\text { Sim } \\
\text { Con } \\
\text { Coo }\end{array}$ & & & & & & \\
\hline Coo & & $\begin{array}{l}\mathrm{Sim} \\
\mathrm{Coo}^{a}\end{array}$ & & $\begin{array}{l}\text { Sim } \\
\text { Con } \\
\text { Coo }\end{array}$ & & & \\
\hline Par & & & $\begin{array}{l}\mathrm{Sim} \\
\mathrm{Coo}^{a}\end{array}$ & & & & \\
\hline $\operatorname{Sim}$ & Sup & $\begin{array}{l}\text { Sim } \\
\text { Con } \\
\text { Coo }\end{array}$ & Par & $\begin{array}{l}\mathrm{Sim} \\
\mathrm{Coo}^{a}\end{array}$ & $\begin{array}{l}\text { Con } \\
\mathrm{Coo}^{a}\end{array}$ & Fun & Qua \\
\hline Con & & & & $\begin{array}{l}\text { Con } \\
\mathrm{COo}^{a}\end{array}$ & $\begin{array}{l}\mathrm{Sim} \\
\mathrm{Coo}^{a}\end{array}$ & & \\
\hline $\begin{array}{l}\text { Fun } \\
\text { Qua }\end{array}$ & & & & & & $\mathrm{Sim}$ & $\mathrm{Sim}$ \\
\hline
\end{tabular}

${ }^{a}$ Coordinates in these cells are not distinguished when the word pairs of highest associative frequency are used exclusively.

to distinguish either one, two, or three tasks from the remainder. Table 4 was derived by testing all cells for all tasks for deviation from the average of the seven matrices. Arithmetic deviations of greater than $10 \%$ were used to define critical cells.

In general, the greatest overlaps occurred in the cells which represent the intersection between the Similars and the task of the given relationship. For example, the greatest overlap for the Superordinates was in cell (Sim, Sup), and this cell was also the critical one for the Superordinates. For Parts the critical cell was (Sim, Par), for Functions (Sim, Fun), and for Qualities (Sim, Qua). Because the cutoff criterion was fairly high, these four relationships had only one critical cell each.

According to Table 4, eleven cells are involved in the discrimination of Coordinates, Similars, and Contrasts, with only two making unique discriminations, (Fun, Fun) and (Qua, Qua). Cells (Sup, Sup), (Coo, Sim), and (Sim, Coo) distinguish only a subset of relationships-a cluster, consisting of Coordinates, Similars, and
Contrasts. In cells (Coo, Coo), (Par, Par), (Sim, Sim) and (Con, Con) this subset is further reduced to include only Similars and Coordinates. Complementary to these are cells (Sim, Con) and (Con, Sim) which group together Coordinates and Contrasts. Finally, cells (Fun, Fun) and (Qua, Qua) isolate Similars to the exclusion of all others. Because of their lack of independence, no cell could distinguish between Contrasts and Coordinates.

\section{Overlap Matrices for Highly Associated Word Pairs}

The method of analysis has imposed some constraint upon the data, which has to be considered in the interpretation of the results. In particular, two factors bear upon the interpretation of the data: (a) the overlap matrices are averages based on 11 word pairs which vary in strength of the relationship; (b) each relationship is composed of a stimulus and its primary response obtained from different Ss. These two factors combine to imply that more pronounced overlap matrices may characterize a more ideal case and hence another analysis was done to check this possibility, as well as to test the importance of the degree of association. 
For each of the seven tasks, the 11 word pairs were ranked according to the frequency of the primary responses given by $S s$ in the second part of the experiment. An example from the Similar relationship will illustrate the procedure. Following are the 11 original stimuli and their primary Similars, with the number of Ss (out of 50 ) who gave the response in the second part of the study: BABY-INFANT, 31; BED-COUCH, 31; TABLEDESK, 26; SHEEP-LAMB, 24; CHAIR-STOOL, 23; LION-TIGER, 22; HAND-PAW, 17; MANBOY, 13; WOMAN-GIRL, 13; HEAD-TOP, 13; FOOT-HAND, 7. Thus for the Similars, the overlap for the highest four pairs is the mean for $B A B Y, B E D, T A B L E$, and SHEEP, while the overlap for the lowest pairs is the mean for MAN, WOMAN, HEAD, and FOOT.

The effect of this division was not systematic for all tasks; the overlap in some cells increases, while it decreases in others. For the Similars, however, every cell on the diagonal increased in magnitude when only the four strongest Similars were considered. This clearly supports the expectation that as word pairs approach ideal similarity, overlaps along the diagonal increase, approaching but never reaching an upper limit of $100 \%$.

\section{Relations among Coordinates, Contrasts, and Similars}

The list of 54 stimuli (see Table 1) reveals a great amount of overlap involving the Coordinates. Empirically there is a lack of independence, particularly between Coordinates and Contrasts, which shared six primary responses, and between Coordinates and Similars which shared two responses. Indeed, one response, FOOT, served as the primary Coordinate, Contrast, and Similar to $H A N D$.

This clearly makes an interpretation of the Coordinate task rather difficult. This lack of independence, however, is not merely an empirical misfortune, but a reflection of logical interrelationships. Apparently, Similars and Contrasts are proper subsets of the Coordinates for which common membership in the class of Superordinates is a necessary requirement. This assumption is supported by the overlap data, particularly for the four word pairs with highest associative frequency. The Superordinate overlap is $81 \%$ for Similars and $79 \%$ for Contrasts. Thus, in giving a Similar or a Contrast, Ss will necessarily respond with a word which is also a Coordinate; in giving a Coordinate, he is likely to respond with a Similar or Contrast.

Empirically, Coordinates, Similars, and Contrasts are characterized by high overlaps along the diagonal cells. However, Similars are clearly separated from Coordinates and Contrasts in certain cells, particularly when the four most highly associated pairs are considered. Thus, all three tasks are characterized by high Superordinate overlap, but Similars show markedly more overlap in the Similar, Contrast, Part, Function, and Quality cells.

\section{A Measure of Similarity}

As mentioned previously, the overlap matrix for a pair of completely similar words is assumed to have $100 \%$ overlap along the diagonal, except in the (Coo, Coo) cell. However, since it was found that Superordinate overlap did not distinguish Similars from Contrasts, only five cells remain significant for similarity: (Par, Par), (Sim, Sim), (Con, Con), (Fun, Fun), and (Qua, Qua). For any pair of words the deviation from $100 \%$ of their response overlap in these five cells can, therefore, be considered as a measure of their similarity. The same measure can also be expressed simply in terms of the mean overlap for two words in these five cells. Since the latter is comparable to previous indexes of associative overlap (Marshall and Cofer, 1963), it was employed here on a sample of 16 word pairs, including the 11. primary Similars. The resulting measures of Restricted-Association Similarity (RAS), ranged from .600 for $B A B Y$ INFANT to .016 for FOOT-FLAT. Other 
word pairs relatively high on the RAS measure were TABLE-DESK (.500), LION-TIGER (.496), CHAIR-STOOL (.492), SHEEP-LAMB (.484), and BEDCOUCH (.408). Some of the pairs in the intermediate range were WOMAN-GIRL (.292), HAND-PAW (.260), HEADBRAIN (.224), MAN-BOY (.204). Lowest in RAS were HEAD-TOP (.068), BABYADULT (.054), and BED-SLEEP (.040). Although $H E A D$ and TOP were primary similars, their RAS was quite low and was exceeded by two of the five Nonsimilar pairs.

Since the relationship between measures of associative overlap and other measures of word similarity would seem to have some relevance for verbal learning (Wimer, 1963), as well as for associative aspects of meaning similarity, a measure of free-associative overlap was taken on 12 of these 16 word pairs to provide a comparison with the results of the restricted association measure. (For four of the word pairs no free-associative data could be found which had been collected on the same population for both words). The measure of free association overlap employed is that of Bousfield, Whitmarsh, and Berkowitz (1960), the index of $\mathrm{Mu}$ tual Relatedness (MR), and is comparable to other measures developed by Cofer (1957) and Deese (1962). MR is simply the number of responses in common to two words, plus their mutual evocations, divided by the total number of responses to the two words. The response frequencies for this index were obtained from the freeassociative norms of Riegel (1965b).

The comparison of the two measures indicated that RAS and MR are less than independent, but by no means identical in what they measure. The range on MR was from .445 for BED-SLEEP to .010 for FOOT-FLAT. In general, word pairs high on RAS were also high on MR, with some significant exceptions, the most striking of which is BED-SLEEP, which ranked fifteenth in RAS but 1st in MR. Likewise FOOT-HAND is higher in MR than RAS, while the reverse is true for some other pairs, including most noticeably $H E A D$ BRAIN, MAN-BOY, WOMAN-GIRL and COUCH-BED. The tau rank-order correlation coefficient for the 12 pairs having both measures is $.24(p<.14)$. Since MR and other measures of free associative overlap have been shown to have predictive value in various verbal learning tasks (Marshall and Cofer, 1963), and since other measures of similarity seem to have less predictive value (Wimer, 1963), it may be of interest to check the predictive value of RAS. Presumably, RAS would be a weaker index of associative interference, since it apparently depends on restricted responses, some of which would not be at high strength, except under restricted conditions.

It would be of more immediate interest, perhaps, to investigate the agreement between this kind of similarity measure and scaled similarity based on Ss' ratings of similarity, as an intuitive validation of the measure. In this regard, it may be significant to note that, as measured by RAS, the most similar word pair is $B A B Y$ INFANT, the only pair of words in the sample approaching real similarity in the usual sense. That is, these words alone (MAN-BOY, WOMAN-GIRL, and HEAD-TOP are possible exceptions) are likely to be interchanged in denoting the same physical object, a fact which gives some intuitive support to the method. Finally, another line of interest in this method lies in the possibility of describing word similarity in terms of dimensions of semantic relationships. Such a procedure would involve an analysis of the response overlaps for the various tasks along lines described by Riegel and Riegel (1963). 


\section{REFERENCES}

Bousfield, W. A., Whitmarsh, G. A., and Berkowitz, H. Partial response identities in associative clustering. J. gen. Psychol., 1960, 63, 233-238.

CoFkn, C. N. Associative commonality and rated similarity of certain words from Haagen's list. Psychol. Rep., 1957, 3, 603-606.

DEESE, J. On the structure of associative meaning. Psychol. Rev., 1962, 59, 421-430.

HaAgen, C. H. Synonymity, vividness, familiarity and association value ratings of 400 pairs of common adjectives. J. Psychol., 1949, 27, 453-463.

Marshale, G. R., and Cofer, C. N. Associative indices as measures of word relatedness: a summary and comparison of ten methods. $J$. verb. Learn. verb. Behav., 1963, 1, 408-421.
Osgood, C. E., Suct, G. J., and Tannenbaum, P. H. The measurement of meaning. Urbana, Ill., Univ. Illinois Press, 1957.

RIEGEL, K. F. The Michigan restricted association norms. Rep. No. 3, NIMH Grant MH 07619 , Univ. Michigan, 1965a.

RuEgel, K. F. Free associative responses to the 200 stimuli of the Michigan Restricted Association Norms. Rep. No. 8, NIMH Grant MH 07619.

Ruecel, K. F., AND RIEGEL, Rutr M. An investigation into denotative aspects of word-meaning. Lang. Speech, 1963, 6, 5-21.

WMER, C. An analysis of semantic stimulus factors in paired-associate learning. J. verb. Learn. verb. Behav., 1963, 1, 397-407.

(Received February 23, 1966) 